\title{
CLOSURE OPERATORS AND GALOIS THEORY IN LATTICES
}

\author{
BY \\ C. J. EVERETT(1)
}

1. Introduction. We study closure operators $\left(A^{*} \supset A, A \subset B^{*}\right.$ implies $\left.A^{*} \subset B^{*}\right)$ on a partially ordered set. Closures in algebra depart from topology in that $(A \cup B)^{*}=A^{*} \cup B^{*}$ fails, and the domain of operation is usually not a boolean algebra, but only a lattice. Closure is strongly related to the concept of the galois correspondence $(\$ 3)$. The idea of general galois correspondences with two basic properties as a method for studying the general galois theory of relations and mathematical structures was first expressed by O. Ore in his Colloquium Lectures in Chicago, $1941[11]\left({ }^{2}\right)$. Every closure arises from a galois correspondence between two partially ordered sets. Every galois correspondence between complete lattices of subsets of two sets is defined by a binary relation between the elements of the sets. Every closure on a complete lattice of subsets of a set is extensible to all subsets. Every closure on all subsets of a set $P$ is defined by a binary relation between $P$ and another set. Every topological closure on a boolean algebra of subsets of a set is extensible to one on all subsets. A generalization of Krull's topology for the automorphism group of an algebraic field is obtained, and applied to characterize the regularly closed subspaces of the conjugate space of a Banach space. A necessary and sufficient condition is given for the existence of a topological closure on all subsets of a group with closure operator $\left(^{*}\right)$ on its subgroup lattice, such that a group is $\left(^{*}\right)$ closed if and only if it is topologically closed. This is combined with results of Baer on primary groups to topologically characterize the subgroups "closed" under the galois correspondence between $P$ and its automorphism group. A metric, totally disconnected topological group may be defined in the automorphism group by using Krull's methods on the subgroups of $P$ of bounded period.

2. Closure operators. Let $\mathfrak{B}$ be a partially ordered set with elements $A, B, C, \cdots$ and order relation (つ). A closure operator (cf. [16]) on $\mathfrak{P}$ is a correspondence $A \rightarrow A^{*}$ on $\mathfrak{B}$ to $\mathfrak{B}$ subject to
C1. $A^{*} \supset A$.
C2'. $A \subset B^{*}$ implies $A^{*} \subset B^{*}$.
C2. $A^{* *}=A^{*}$.
C3. $A \subset B$ implies $A^{*} \subset B^{*}$.

Presented to the Society, September 13, 1943; received by the editors May 29, 1943.

(1) The author is deeply indebted to Professor Oystein Ore for the privilege of reading parts of his unpublished manuscript for the Colloquium Lectures while Sterling Fellow at Yale, 1941. This indebtedness is heaviest in $\$ 3$ where the concepts, notation, and terminology are due to Ore.

(2) Numbers in brackets refer to the bibliography at the end of the paper. 
It is trivial to verify the equivalence of $\mathrm{C} 1, \mathrm{C} 2$ ' with $\mathrm{C} 1-3$.

In case $\mathfrak{B}$ is a lattice (union $V$, intersection $\cap$ ) a closure operator may satisfy one or more of the additional properties

C4. $(A \bigvee B)^{*}=A^{*} \bigvee B^{*}$.

C5. $0^{*}=0$ (if $\mathfrak{B}$ contains a zero: $0 \subset A$, all $A \in \mathfrak{B}$ ).

C6. $P, Q$ minimal over 0 (points) and $P^{*}=Q^{*}$ implies $P=Q$.

C7. For every point $P, P^{*}=P$.

C8. $(A \cap B)^{*}=A^{*} \cap B^{*}$.

Denote by $\left(5\right.$ the class of all "closed" elements $C$ (for which $C^{*}=C$ ).

THEOREM 1. If $\mathfrak{B}$ is a partially ordered set with a $\mathrm{C} 1-3$ closure $\left(^{*}\right)$, then the set $\mathbb{S}$ of closed elements has the properties:

(51. If $C_{\alpha}$ are elements of $\mathbb{( S}$ and $\cap C_{\alpha}$ exists in $\mathfrak{B}$ then $\cap C_{\alpha}$ is in $\mathbb{S}$ and is $a$ g.l.b. of the $C_{\alpha}$ relative to $\mathbb{E}$.

(52. If $C_{\alpha}$ are elements of $\mathbb{S}$ and $\vee C_{\alpha}$ exists in $\mathfrak{B}$ then $\left(\vee C_{\alpha}\right)^{*}$ is in $\mathbb{S}$ and is a l.u.b. of the $C_{\alpha}$ relative to $\mathbb{E}$.

(53. If $\left\{C_{\alpha}\right\}$ is the set of all $C \in \mathbb{S}$ for which $C \supset A$, then $\cap C_{\alpha}$ exists in $\mathfrak{B}$ and is $A^{*}$.

COROLlaRY 1. If $\mathfrak{B}$ is a (conditionally) complete lattice, so is $\mathbb{S}$ under the same order.

Corollary 2. If $X \rightarrow X^{*}$ is a $\mathrm{C} 1-3$ closure on the lattice (boolean algebra) $\mathfrak{B}$ of all subsets of a set, then the closed sets form a complete lattice.

The proofs are trivial. For example (53: If $A \subset C_{\alpha}$, then $A^{*} \subset C_{\alpha}{ }^{*}=C_{\alpha}$, and $A^{*}$ is a lower bound of the $C_{\alpha}$. However, if $B \subset$ every $C_{\alpha}, A^{*}$ is itself a $C_{\alpha} \supset A$, and $B C A^{*}$.

We may even begin, following E. H. Moore [10], with a set of 'undefined "closed" elements and define $A^{*}$ by means of them, thus:

THEOREM 2. If $\mathfrak{B}$ is a partially ordered set, with unit 1 , and a subset $\mathbb{E}$ containing 1 and containing, for each $A \in \mathfrak{B}$, the intersection of all $C \supset A, C \in \mathbb{\mho}$, then the definition $A^{*}=\cap(C ; C \in \mathbb{E}, C \supset A)$ is a C1-3 closure.

Proof. C1 is trivial. Since $A^{*}$ is in $\sqrt{ }$, $A^{* *}=\cap\left(C ; C \in \mathbb{S}, C \supset A^{*}\right) \subset A^{*}$. If $A \subset B$, the set $(C ; C \in \mathbb{C}, C \supset B)$ is a subset of $(C ; C \in \mathbb{E}, C \supset A)$. Hence $A^{*} \subset B^{*}$.

The concept of closure introduced here departs from its topological source in two directions. The domain of the operator need not be the boolean algebra of all subsets of a set, or indeed a boolean algebra at all; and moreover, the restrictive properties $\mathrm{C} 4, \mathrm{C} 6, \mathrm{C} 7$ are not assumed. There seems some reason for hoping that a highly developed theory of such operators may throw light on many algebraic structures which are usually lattices rather than boolean algebras, and admitting closures satisfying $\mathrm{C} 3$ rather than $\mathrm{C} 4$.

Example 1. $\mathfrak{B}$ is the boolean algebra of all subsets of a set, $A^{*}$ the topological closure of subset $A$; properties C1-7 hold. 
Example. 2. $\mathfrak{B}$ is the boolean algebra of all subsets $A$ of a group, $A^{*}$. is the subgroup generated by elements of $A ; \mathrm{C} 1-3$ hold, but not $\mathrm{C} 6,7,4$.

Example 3. $\mathfrak{B}$ is the boolean algebra of all subsets $A$ of the quotient field $\mathfrak{F}$ of domain of integrity $\mathfrak{D}, A^{*}$ the general Prüfer ideal generated by the elements of $A$. (A Prüfer ideal [12] is defined as a class $\{A\} \subset \mathfrak{F}$ associated with the subset $A \subset \mathfrak{F}$, subject to $A \subset\{A\} ; B \subset\{A\}$ implies $\{B\} \subset\{A\} ;$ single element $\alpha\}=$ principal ideal $\mathfrak{D} \alpha ; \alpha \in\{A\}$ implies $\alpha \beta \in\{A \beta\})$. $\mathfrak{B}$ satisfies $\mathrm{C} 1-3$. Thus Dedekind ideals, $\mathfrak{B}$-ideals, $\mathfrak{A}$-ideals are examples.

Example $4 . \mathfrak{B}$ is the lattice of positive integers $A$, with $A \supset B$ meaning " $A$ divides $B$." Define $A$ * as the product of all the distinct prime factors of $A$. One verifies $C 1-4, C 8$ ! There are no points in $\mathfrak{B}$.

Example $5 . \mathfrak{B}$ is the boolean algebra of subsets $A$ of the unit square, $A^{*}$ the "direct product" of the projections $A_{x}$ and $A_{y}$ of $A$. More generally, the projective boolean algebras of Ulam [15] are examples. One verifies C1-3, C7, not C4.

Example 6. $\mathfrak{B}$ is lattice modul $[6 ;$ p. 105], with 0 the zero of addition, $A^{*}=A \bigvee 0$, the positive part of $A ; \mathrm{C} 1-4$ hold.

As we shall see in the next section, all closure operators on partially ordered sets arise, many in a natural manner, from the general galois correspondences. We defer further examples to that place.

3. Galois correspondences. We now summarize Ore's theory $\left(^{(1)}\right.$. Let $\mathfrak{B}, \mathfrak{Q}$ be partially ordered sets, admitting a pair of correspondences $(\gamma), P \rightarrow \mathfrak{Q}(P)$ on $\mathfrak{B}$ to $\mathfrak{Q}$, and $Q \rightarrow \mathfrak{B}(Q)$ on $\mathfrak{Q}$ to $\mathfrak{B}$, subject to

G1. $P_{1} \supset P_{2}$ implies $\mathfrak{Q}\left(P_{1}\right) \subset \mathfrak{Q}\left(P_{2}\right)$, and $Q_{1} \supset Q_{2}$ implies $\mathfrak{B}\left(Q_{1}\right) \subset \mathfrak{P}\left(Q_{2}\right)$.

G2. $\mathfrak{B Q}(P) \supset P$, and $\mathfrak{Q}(Q) \supset Q$.

ThEOREM 3 (ORE). The operator $P \rightarrow P^{*}=\mathfrak{B Q}(P)$ is a closure satisfying C1-3. The element $P$ is closed $\left(P=P^{*}\right)$ if and only if $P=\mathfrak{B}(Q)$ for some $Q \in \mathfrak{Q}$. If $\mathfrak{B}, \mathfrak{Q}$ are complete ordered, the correspondences $(\gamma)$ define dual isomorphisms of the lattices of closed elements, and $\mathfrak{P}\left(\vee Q_{\alpha}\right)=\cap \mathfrak{P}\left(Q_{\alpha}\right)$.

Verification of $\mathrm{C} 1, \mathrm{C} 3$ is trivial. For $\mathrm{C} 2$, note that $\mathfrak{Q} \mathfrak{P Q}(P)=\mathfrak{Q}(P)$. For $P \subset \mathfrak{P Q}(P)$ implies $\mathfrak{Q}(P) \supset \mathfrak{Q} \mathfrak{B Q}(P)$ while $\mathfrak{Q} \mathfrak{B}(\mathfrak{Q} P) \supset \mathfrak{Q} P$. The condition for $P$ closed follows immediately. Complete order in $\mathfrak{P}, \mathfrak{Q}$ implies the same for their lattices of closed elements by Corollary 1. Cf. [6; p. 25]. Finally, $\vee Q_{\alpha} \supset Q_{\alpha}$ implies $\mathfrak{B}\left(\vee Q_{\alpha}\right) \subset \mathfrak{B}\left(Q_{\alpha}\right)$, and $\mathfrak{P}\left(\vee Q_{\alpha}\right) \subset \cap \mathfrak{B}\left(Q_{\alpha}\right)$. But $\cap \mathfrak{B}\left(Q_{\alpha}\right)$ $\subset \mathfrak{P}\left(Q_{\alpha}\right)$ implies $\mathfrak{Q}\left(\cap \mathfrak{B} Q_{\alpha}\right) \supset \mathfrak{Q} \mathfrak{B}\left(Q_{\alpha}\right) \supset Q_{\alpha}, \mathfrak{Q}\left(\cap \mathfrak{B} Q_{\alpha}\right) \supset \vee Q_{\alpha}$, and $\cap \mathfrak{B}\left(Q_{\alpha}\right)$ $\subset \mathfrak{P Q}\left(\cap \mathfrak{B Q} Q_{\alpha}\right) \subset \mathfrak{P}\left(\vee Q_{\alpha}\right)$.

Ore calls a $G 1,2$ correspondence $(\gamma)$ a galois correspondence, and under such, $\mathfrak{B Q}(P)$ the galois closure of $P$.

The equivalence of the ideas of closure and galois closure is emphasized by the following theorem.

Theorem 4. If $\mathfrak{P}$ is a partially ordered set with $\mathrm{C} 1-3$ closure $P \rightarrow P^{*}$ and 
closed elements $\mathfrak{S}$, and $\mathfrak{Q}$ is the partially ordered set of all subsets $Q$ of $\mathfrak{C}$ whose intersections exist in $\mathfrak{B}$, then the correspondences $(\gamma): P \rightarrow \mathfrak{Q}(P)=($ all $C \in \mathfrak{C}$; $C \supset P$ ) and $Q \rightarrow \mathfrak{B}(Q)=\cap($ all elements of $Q)$ are galois $\mathrm{G} 1,2$, and $P^{*}=\mathfrak{B Q}(P)$. Thus every closure is a galois closure.

Proof. G1. That $P_{1} \supset P_{2}$ implies $\mathfrak{Q}\left(P_{1}\right) \subset \mathfrak{Q}\left(P_{2}\right)$ is trivial; $Q_{1} \supset Q_{2}$ implies $\cap\left(Q_{1}\right) \supset \cap\left(Q_{2}\right)$, since $\cap\left(Q_{1}\right) \subset$ Cevery element of $Q_{1}$, hence of $Q_{2} . \mathscr{Q}(P)$ is in $\mathscr{Q}$ by $\$ 3$ of Theorem 1 .

G2. $\mathfrak{P Q}(P)=\cap(C ; C \in \mathbb{E}, C \supset P)=P^{*} \supset P$ by $\mathbb{E} 3$ of Theorem 1 and $\mathrm{C} 1$. Also $\mathfrak{Q P}(Q)=(C ; C \in \mathbb{C}, C \supset \cap(Q)) \supset Q$, for every element of $Q$ is in $\mathbb{C}$ and $\supset \cap(Q)$.

Example 7. Let $E$ be a set, $\mathfrak{B}$ the lattice of all partitions (equivalence relations) of the elements of $E ; P_{1} \supset P_{2}$ means $P_{1}$ is a refinement of $P_{2} ; \mathscr{Q}$ is the lattice of all subgroups of the full symmetric group $S$ (all 1-1 transformations) of $E$ onto $E$. Let $\mathfrak{P}(Q)$ be the partition defined by the equivalence relation $a=b$ meaning $a=b^{\gamma}$ for some $\gamma \in Q$, and $\mathfrak{Q}(P)$ the group leaving the classes of $P$ undisturbed. This is a galois correspondence. Indeed G. Birkhoff [5] proves (a) every partition is galois closed, and $\mathfrak{Q}\left(\vee P_{\alpha}\right)=\cap \mathfrak{Q} P_{\alpha}$ (see Theorem 3), also (b) $\mathfrak{Q}\left(P_{1} \cap P_{2}\right)=\mathfrak{Q}\left(P_{1}\right) \vee \mathfrak{Q}\left(P_{2}\right)$. Thus the partition lattice $\mathfrak{B}$ is isomorphic to the lattice $\mathfrak{S}(\mathfrak{Q})$ of "closed" subgroups of $\mathfrak{S}$.

However, most examples of closure operators arising naturally from galois correspondences involve $\mathfrak{B}$ 's which are lattices of subsets of a set. We turn now to these.

4. Galois correspondences for lattices of subsets. G. Birkhoff $[6 ;$ p. 24] and $O$. Ore [11] have studied this case. We shall attempt to show the relationship between their viewpoints.

Birkhoff considers galois correspondences arising from a binary relation $\rho$ between the elements of two sets $\Pi$ and $\Omega$, so that for every pair $p \in \Pi, q \in \Omega$ either $p \rho q$ or not. For every subset $P \subset \Pi$ define $\Omega_{p}(P)=(q ; q \in \Omega ; p \rho q$, all $p \in P)$, and for $Q \subset \Omega$ define $\mathfrak{B}_{\rho}(Q)=(p ; p \in \Pi ; p \rho q$, all $q \in Q)$. These are galois correspondences for the lattices $\mathfrak{B}, \mathfrak{Q}$ of all subsets $P, Q$ of $\Pi, \Omega$ respectively.

Ore, in his work on relations, begins from the following standpoint. Let $\Pi, \Omega$ be sets, $\phi(\Pi), \psi(\Omega)$ families of subsets $P \subset \Pi, Q \subset \Omega$ respectively. A galois correspondence is defined as any dual mapping $P \rightarrow \mathfrak{Q}(P)$ on $\phi(\Pi)$ to $\psi(\Omega)$ and $Q \rightarrow \mathfrak{P}(Q)$ on $\psi(\Omega)$ to $\phi(\Pi)$, subject to $\mathrm{G} 1,2$.

THEOREM 5. Every Birkhoff correspondence defines an Ore correspondence. Conversely, if the families $\phi(I I), \psi(\Omega)$ of an Ore correspondence are closed under (unrestricted) union and intersection, and cover $\Pi, \Omega$ respectively, then the Ore correspondence is defined by a binary relation between $\Pi$ and $\Omega$.

The first statement is immediate, using for $\phi(\Pi), \psi(\Omega)$ the families of all subsets of $\Pi, \Omega$ respectively.

Suppose now an Ore correspondence is given between families $\phi(\Pi)$ and 
$\psi(\Omega)$ as stated. Define the binary relation $p \rho q$ between $\Pi$ and $\Omega$ to mean that there exists a $Q \in \psi(\Omega)$ such that $p \in \mathfrak{B}(Q)$ and $q \in \mathfrak{Q} \mathfrak{B}(Q)$. This binary relation defines the Birkhoff correspondences $\mathfrak{B}_{\rho}(Q)$ and $\mathfrak{Q}_{\rho}(P)$ for all subsets $P \subset \Pi, Q \subset \Omega$. We need only verify that $\mathfrak{B}(Q)=\mathfrak{B}_{\rho}(Q)$ and $\mathfrak{Q}(P)=\mathfrak{Q}_{\rho}(P)$ for $P \in \phi(\Pi), Q \in \psi(\Omega)$. First, $\mathfrak{Q}(P) \subset \mathfrak{Q}_{p}(P)$. For let $q \in \mathfrak{Q}(P)$ and $p \in P$. Then $\mathfrak{Q}(P) \in \psi(\Omega), p \in P \subset \mathfrak{B} \mathfrak{Q}(P)$ and $q \in \mathfrak{Q} \mathfrak{B} \mathfrak{Q}(P)=\mathfrak{Q}(P)$. Hence $p \rho q$ for all $p \in P$. Second, $\mathfrak{Q}_{p}(P) \subset \mathfrak{Q}(P)$. For, let $q \in \mathfrak{Q}_{\rho}(P)$. For every $p_{\alpha} \in P$, there exists a $Q_{\alpha} \in \psi(\Omega)$ such that $q \in \mathfrak{Q} \mathfrak{B}\left(Q_{\alpha}\right)$ and $p_{\alpha} \in \mathfrak{B}\left(Q_{\alpha}\right)$. Then $q \in \cap \mathfrak{Q} \mathfrak{B}\left(Q_{\alpha}\right)$ $=\mathfrak{Q}\left(\cup \mathfrak{B} Q_{\alpha}\right)$. But $P=\cup_{p_{\alpha}} \subset \cup \mathfrak{B} Q_{\alpha}$, hence $q \in \mathfrak{Q}\left(\cup \mathfrak{B} Q_{\alpha}\right) \subset \mathfrak{Q}(P)$.

Proof that $\mathfrak{B}(Q)=\mathfrak{B}_{p}(Q)$ for $Q \in \psi(\Omega)$ involves no new difficulty and is even simpler.

Remark. The latter half of Theorem 5 holds even if the sets $\phi(\Pi), \psi(\Omega)$ are complete ordered lattices of sets $P, Q$ under set inclusion, with set intersection effective as g.l.b.

Theorem 6. $A$ C1-3 closure operator defined on a complete lattice $\mathfrak{B}=\phi(\Pi)$ of subsets $P$ of a set $\Pi$ which covers $\Pi$ and has set intersection for $(\cap)$ can be extended to a C1-3 closure on all subsets of $\Pi$.

Let $P \rightarrow P^{*}$ be the closure on $\mathfrak{B}$. Define for every set $X \subset \Pi$ the set $X \dagger=\cap\left(P \in \mathfrak{P}, P^{*}=P, P \supset X\right)$, which is a closure by Theorem 2 .

Theorem 7. Every C1-3 closure on all subsets $P$ of a set $\Pi$ is the galois closure defined by a binary relation between $\Pi$ and a suitable set $\Omega$.

Define $\Omega$ as the class of all closed sets $C$ of $\Pi$. Define $p \rho C$ between the elements $p \in \Pi$ and $C \in \Omega$ to mean $p \in C$. We must prove $P^{*}=\mathfrak{B}_{p} \mathfrak{Q}_{p}(P)$. Now $\mathfrak{Q}_{p}(P)=(C ; C \in \Omega ; p \in C$, all $p \in P)=(C ; C \in \Omega, C \supset P)$, and $\mathfrak{P}_{\rho} \mathfrak{Q}_{\rho}(P)=(p \in \Pi$, $p \in$ all $C \supset P)=\cap(C ; C \in \Omega, C \supset P)=P^{*}$ by $₫ 3$ of Theorem 1 .

Corollary 3. Every $\mathrm{C} 1-3$ closure relation $P^{*}$ on a complete ordered set $\mathfrak{B}$ of subsets of a set $\Pi$ which covers $\Pi$ is defined by the galois closure $\mathfrak{B}_{\rho} \mathfrak{Q}_{\rho}(P)$ of. a binary relation between $\Pi$ and a suitable set $\Omega$.

Theorem 8. A C1-4 closure operator $\left({ }^{*}\right)$ defined on a complete boolean algebra $\mathfrak{P}=\phi(\Pi)$ of subsets. of a set covering $\Pi$ (set union and set intersection) can be extended to a C1-4 closure operator on all subsets of $\Pi$.

We need only verify that $X \dagger=n\left(C ; C \in \mathfrak{B} ; C^{*}=C, C \supset X\right)$ satisfies C4. For $X, Y \subset \Pi,(X \cup Y) \dagger \subset X \dagger \cup Y \dagger$. For let $z \in(X \cup Y) \dagger$, but not to $X \dagger \cup Y \dagger$. Then there exist closed sets $C_{1} \supset X, C_{2} \supset Y$ with $z \notin C_{1}, z \notin C_{2}$. But C4 holds in $\mathfrak{B}$, and $\left(C_{1} \cup C_{2}\right)=\left(C_{1} \cup C_{2}\right) *$ is closed and contains $X \cup Y$. Since $z \in(X \cup Y) \dagger$, $z \in C_{1} \cup C_{2}$, we have a contradiction.

Thus the topological closures on boolean algebras of restricted subsets of $\Pi$ are realized by ordinary topologies of $\Pi$ (cf. Terasaka [14]).

Remark. Since the MacNeille completion [9] embeds a partially ordered 
set $\mathfrak{P}$ in a complete ordered set of subsets of $\mathfrak{B}$ with preservation of order and (unrestricted) intersection and union, it is clear that every $\mathrm{C} 1-3$ closure on $\mathfrak{P}$ is essentially a C1-3 closure on a partially ordered set of subsets of a set, and hence extensible to all subsets of that set.

5. Krull topologization. We now phrase a question of the following sort. Given a family $\Omega=\psi(\Omega)$ of subsets $Q$ of a set $\Omega$ on which a C1-3 closure (*) is defined, under what circumstances is it possible to define a Hausdorff topology in $\Omega$ in such way that a set $Q \in \Omega$ is $\left({ }^{*}\right)$ closed if and only if it is topologically closed. In this section we give a partial answer suggested by the remarkable paper of Krull [8].

Example 8 (Krull). Let $\Omega=\{a\}$ be an algebraic field over $\Omega_{0}, \Omega$ the group of all its $\Omega_{0}$-isomorphisms $q$, and the binary relation $a \rho q$, the condition $a q=a$, between $\Omega$ and $\Omega$. One defines $\Omega_{\rho}(Q)$ and $\Omega_{\rho}(K)$ for $Q$ a subset of $\Omega, K$ a subset of $\Omega$ in the usual way (cf. $\S 4$ ). A set $K$ is galois-closed, $K=\Omega_{\rho} \Omega_{p}(K)$, if and only if it is a field. If a subset $Q \subset \Omega$ is galois-closed, $Q=\Omega_{\rho} \Omega_{\rho}(Q)$, it is a subgroup, but not every subgroup is closed. We may regard $\mathfrak{Q}$ as the subgroup lattice of $\Omega$ and $Q \rightarrow Q^{*}=\Omega_{\rho} \Omega_{\rho}(Q)$ a closure operator on $Q$. Krull shows how a Hausdorff topology may be defined for the elements (points) of $\Omega$ so that a group $Q$ of $\mathfrak{Q}$ is galois closed if and only if it is topologically closed.

We couch Krull's essential idea in the following general way:

THEOREM 9. Let $\mathfrak{B}$ be a complete lattice with unit $1, \mathfrak{Q}$ the lattice of all subgroups $Q$ of a group $\Omega=\{q\}$, additively written, admitting a galois correspondence $\mathrm{G} 1,2$ with $\mathfrak{Q}(1)=0$. Suppose $\mathfrak{B}$ contains a family $\mathfrak{F}$ of elements $F$ such that

F1. For $F_{1}, F_{2} \in \mathfrak{F}$, one has $F_{1} \bigvee F_{2} \in \mathfrak{F}$.

$\mathfrak{F} 2$. Every $P \in \mathfrak{B}$ is a union $\bigvee F_{\alpha}$ of $F_{\alpha}$ 's in $\mathfrak{F}$.

For every $F \in \mathfrak{F}, q \in \Omega$, define the coset $q+\mathfrak{Q}(F)$ as a neighborhood of $q$. Then $\Omega$ is a Hausdorff space (topological group if $\mathfrak{Q}(F)$ is invariant for all $F \in \mathfrak{F})$.

If $\mathfrak{F}$ has the further property

F3. For every $F \in \mathfrak{F}, Q \in \mathfrak{Q}$, one has $\mathfrak{Q} \mathfrak{P}(Q) \subset Q+\mathfrak{Q}(F)$, the set of all sums $q_{1}+q_{2}, q_{1} \in Q, q_{2} \in \mathfrak{Q}(F)$, then the subgroup $Q$ is galois closed if and only if it is closed in the neighborhood topology.

I. $q \in q+\mathfrak{Q}(F)$.

II. $q^{\prime} \in q+\mathfrak{Q}(F)$ implies $q^{\prime}+\mathfrak{Q}(F)=q+\mathfrak{Q}(F)$.

III. $\left(q+\mathfrak{Q}\left(F_{1}\right) \cap\left(q+\mathfrak{Q}\left(F_{2}\right)\right)=q+\left(\mathfrak{Q}\left(F_{1}\right) \cap \mathfrak{Q}\left(F_{2}\right)\right)=q+\mathfrak{Q}\left(F_{1} \vee F_{2}\right)\right.$.

IV. For $q_{1} \neq q_{2}$ there exists an $F \in \mathfrak{F}$ such that $q_{1}+\mathfrak{Q}(F)$ and $q_{2}+\mathfrak{Q}(F)$ are disjoint. For, if for every $F$ the neighborhoods intersect, one has $-q_{2}+q_{1}$ $\in \mathfrak{Q}(F)$, all $F$. But $0=\mathfrak{Q}(1)=\mathfrak{Q}\left(\vee F_{\alpha}\right)=\cap \mathfrak{Q} F_{\alpha}$ contains $-q_{2}+q_{1} \neq 0$, which is a contradiction.

V. For every $F, q_{1}+q_{2}+\mathfrak{Q}(F)=q_{1}+\mathfrak{Q}(F)+q_{2}+\mathfrak{Q}(F)$ for $\mathfrak{Q}(F)$ invariant.

VI. For every $F,-(q+\mathfrak{Q}(F))=-q+\mathfrak{Q}(F)$ for $\mathfrak{Q}(F)$ invariant. 
Thus the axioms are established for a topological group.

VII. Let $Q$ be galois closed. Since then $Q=\mathfrak{Q}(P)$ for some $P$, namely $\mathfrak{B}(Q)$, we show that every $\mathfrak{Q}(P)$ is closed in the Krull topology. Let $q$ be a limit point of $\mathfrak{Q}(P)$, and $P=\bigvee F_{\alpha}, F_{\alpha} \in \mathfrak{F}$. For every $F_{\alpha}$ there is a $q_{\alpha} \in \mathfrak{Q}(P)$ $\subset \mathfrak{Q}\left(F_{\alpha}\right)$ such that $q_{\alpha} \in q+\mathfrak{Q}\left(F_{\alpha}\right)$. Thus $q \in \mathfrak{Q}\left(F_{\alpha}\right)$, all $F_{\alpha}$. But $\mathfrak{Q}(P)$ $=\mathfrak{Q}\left(\bigvee F_{\alpha}\right)=\cap \mathfrak{Q}\left(F_{\alpha}\right)$ contains $q$. Hence $q \in \mathfrak{Q}(P)$ and $\mathfrak{Q}(P)$ is topologically closed.

We use $\mathfrak{F} 3$ only in the final step.

VIII. Suppose $Q$ is topologically closed. We prove $q \in \mathfrak{Q} \Re Q$ implies $q$ a limit point of $Q$. One has for every $F, \mathfrak{Q} Q \subset \subset Q+\mathfrak{Q}(F), q=q_{1}+q_{2}, q_{1} \in Q$, $q_{2} \in \mathfrak{Q}(F)$. Hence $q_{1} \in q+\mathfrak{Q}(F), q_{1} \in Q$.

Corollary 4. Under the conditions $\mathfrak{F 1 , 2}$ of Theorem 9, the Krull topology is zero-dimensional and hence totally disconnected.

For every neighborhood $\mathfrak{Q}(F)$ of the zero is closed.

TheOREM 10. If the set $\mathfrak{F} 1,2$ of Theorem 9 contains a denumerable sequence $F_{1} \subset F_{2} \ldots$ such that every $F$ is covered by some $F_{n} \supset F$ then the Krull topology is metrizable.

For every $q \in \Omega, n=1,2, \cdots$ define $U_{n}(q)=q+\mathfrak{Q}\left(F_{n}\right)$. Since every neighborhood $U(q)$ is of the form $q+\mathfrak{Q}(F)$ and $F \subset F_{n}$ for some $n, U(q) \supset U_{n}(q)$. The $U_{n}(q)$ form a basis for the Krull space, and $U_{n+1}(q) \subset U_{n}(q)$. Also, for every $q \in \Omega, n=1,2, \cdots$, there is an $m=m(q, n)=n$ itself, such that $U_{m}(q) \cap U_{m}\left(q^{\prime}\right)$ non-void implies $U_{m}\left(q^{\prime}\right) \subset U_{n}(q)$. For if cosets $q+\mathscr{Q}\left(F_{m}\right)$ and $q^{\prime}+\mathfrak{Q}\left(F_{m}\right)$ intersect, they coincide, and $U_{m}\left(q^{\prime}\right)=U_{n}\left(q^{\prime}\right)=U_{n}(q)$. The metrization follows from the theorem of Frink [7]. The metric is simply $\delta\left(q, q^{\prime}\right)=1 / n$ where $n$ is the first $m$ for which $q+\mathfrak{Q}\left(F_{m}\right)$ and $q^{\prime}+\mathfrak{Q}\left(F_{m}\right)$ are disjoint.

The application to Example 8 of algebraic fields is of course clear. The $F$ 's are simply the finite algebraic subfields of $\Omega$. However, we proceed to apply Theorem 9 to the linear functional space of a Banach space.

6. On Banach spaces. Let $I I$ be a Banach space, and $\Omega$ the space of all linear functionals $q$ on $\Pi$ to the real field. Consider the binary relation $p \rho q$ between $\Pi$ and $\Omega$ defined by $q(p)=0$. This defines a galois correspondence between the subsets of $\Pi$ and those of $\Omega$. $P \rightarrow \mathfrak{Q}_{p}(P), Q \rightarrow \Re_{p}(Q), P \subset \Pi, Q \subset \Omega$. Banach $[3$, p. 57] proves that for every norm-closed subspace $P \subset \Pi$ and element $p \notin P$, there is a linear functional $q$ such that $q(P)=0$ but $q(p)=1$. It follows that a subset $P \subset \Pi$ is galois-closed, $P=\mathfrak{B}_{p} \mathfrak{Q}_{p}(P)$, if and only if $P$ is a norm-closed subspace. However, it is not true that every closed subspace $Q$ of $\Omega$ is galois closed, $\mathfrak{Q}_{p} \mathfrak{B}_{p}(Q)=Q$ (cf. Banach loc. cit. p. 116). The subspaces $Q$ of $\Omega$ which are galois closed are called regularly closed by Banach.

Banach shows that a subspace $Q$ of $\Omega$ is regularly closed if and only if it is closed in a topology of transfinite sequences, and for $\Pi$ II separable, if and only 
if it is closed in the topology of weak convergence in $\Omega$. Alaoglu [1] has recently characterized regular closure in terms of Moore-Smith convergence.

We shall show how a Krull topology may be introduced into $\Omega$ in such a way that a subspace $Q$ of $\Omega$ is galois (that is, regularly) closed if and only if it is closed in the Krull topology. Separability is not assumed, and the Krull topology defines a topological group on $\Omega$.

Our topology is closely related to that of Birkhoff [4] for general Hausdorff spaces, but differs in that our space $\Omega$ is not a transformation group of $\Pi$.

Theorem 11. The set $\mathfrak{B}$ of all subspaces of a Banach space $\Pi$ is a complete lattice with unit $\Pi$, and the set $\mathfrak{Q}$ of all subspaces of the linear functional space $\Omega$ admits with $\mathfrak{P}$ a galois correspondence determined by the relation $q(p)=0$, jor $p \rho q$; also $\mathfrak{Q}(\Pi)=0$. $\mathfrak{B}$ contains the family $\mathfrak{F}$ of all finite dimensional subspaces, of $\Pi$, and these satisfy $\mathfrak{F 1 - 3 .}$ A subspace $Q$ of $\Omega$ is galois closed (that is, regularly closed) if and only if it is closed in the Krull topology of Theorem 9. There is a lattice isomorphism between the norm-closed subspaces of $\Pi$ and the regularly closed subspaces of $\Omega$.

We need only verify $\mathfrak{F} 3$. Let $F$ be a finite dimensional subspace of $\Pi$, with basis $x_{1}, \cdots, x_{n}, Q$ any subspace of $\Omega$. Then $F \cap \Re(Q)$ is a subspace of $F=\left(x_{1}, \cdots, x_{n}\right)$. Every linear functional $q$ of $\Omega$ defines a linear functional $q_{F}$ on $F$ to $R$. Hence $Q$ defines a space $Q_{F}$ of linear functionals on $F$ to $R$. If $q \in \mathbb{Q}(F \cap \Re(Q))$, then $q_{F}(F \cap \mathfrak{B}(Q))=0$. But $F \cap \Re(Q)$ is precisely the subspace of $F$ annihilated by all functionals of $Q_{F}$. Hence $q_{F} \in Q_{F}$, since for finite dimensional spaces the orthogonal complement is idempotent. Thus there exists a $q^{\prime} \in Q$ such that $q$ and $q^{\prime}$ have the same effect on $F$. Thus $-q^{\prime}+q$

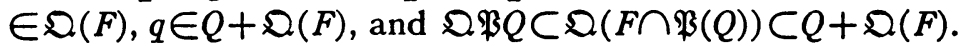

COROllary 5. Every infinite dimensional subspace $Q$ of $\Omega$ is dense in itself. Hence every regularly closed such subspace is perfect (in the Krull topology).

Let $Q$ be $\infty$-dimensional. It suffices to show that for every $F \in \mathfrak{F}$ there is a $q \neq 0$ of $Q$ in $0+\mathfrak{Q}(F)$, that is, $Q \cap \mathfrak{Q}(F) \neq 0$. If $Q_{F}$ denotes the space of all linear functionals defined on $F$ by elements of $Q$, one has $Q_{F} \simeq Q / Q \cap Q(F)$. Since $Q_{F}$ is finite dimensional, and $Q \infty$-dimensional, $Q \cap \mathfrak{Q}(F) \neq 0$ (cf. Krull [8]).

Although the Krull topology and that of weak convergence serve equally well to characterize the regularly closed subspaces of $\Omega$ for separable $\Pi$, they are not equivalent topologies since the latter is not a Hausdorff topology: $Q^{* *} \neq Q^{*}$ in general (cf. Banach $[3$, p. 209]).

While Theorem 9 provides a partial answer to the general question raised at the beginning of $\$ 5$, which covers infinite algebraic number fields and Banach spaces, it is apparently rather special in its use of the family $\mathfrak{F}$.

We shall see later that the condition $\mathfrak{F} 3$ fails in the case of primary abelian 
groups, and that while the Krull topology is of some interest in itself, it fails to characterize the galois closed subgroups.

7. A necessary and sufficient condition. Let $\Omega$ be a group on whose subgroup lattice $\mathfrak{Q}$ is defined a C1-3 closure $Q \rightarrow Q^{*}$.

THEOREM 12. In order that there exist a C1-4 closure $X \rightarrow X \dagger$ on the subsets of $\Omega$ such that, for a subgroup $Q$,

(a) $Q \dagger$ is a subgroup of $\Omega$,

(b) $Q \dagger=Q$ if and only if $Q^{*}=Q$,

it is necessary and sufficient that,

(c) for groups $Q, Q_{1}, \ldots, Q_{n}, Q \subset Q_{1}{ }^{*} \cup \ldots \cup Q_{n}{ }^{*}$ implies $Q^{*} \subset Q_{1}{ }^{*} \cup \ldots$ $\cup Q_{n}^{*}$.

Necessity. We note first that for a group $Q, Q^{*}=Q \dagger$. Since $Q^{*}$ is a group and $Q^{* *}=Q^{*}$, from (b), $Q^{*} \dagger=Q^{*} \supset Q$ and $Q^{*}=Q^{*} \dagger \supset Q \dagger$. Since by (a), $Q \dagger$ is a group, and $Q \dagger \dagger=Q \dagger$, from (b) $Q \dagger^{*}=Q \dagger \supset Q$. Thus $Q \dagger=Q \dagger^{*} \supset Q^{*}$. Hence if $Q \subset Q_{1}^{*} \cup \ldots \cup Q_{n}^{*}$, we have $Q^{*}=Q \dagger \subset\left(Q_{1}^{*} \cup \ldots \cup Q_{n}^{*}\right) \dagger=Q_{1}^{*} \dagger \cup \ldots$ $\cup Q_{n}{ }^{*} \dagger=Q_{1}{ }^{*} \cup \ldots \cup Q_{n}{ }^{*}$.

Sufficiency. Define $X \dagger=\cap$ (all finite set unions $\left.\sum_{1}^{n} Q_{i}^{*} \supset X\right)$ ). Clearly $X \dagger \supset X$. Moreover $X \dagger \dagger=\bigcap$ (all finite $\left.\sum Q_{i}{ }^{*} \supset X \dagger\right) \subset X \dagger$ since all the $\sum Q_{i}{ }^{*} \supset X$ surely contain $X \dagger$. Finally, $\mathrm{C} 3$ is trivial, and $(X \cup Y) \dagger=\cap$ (finite $\sum Q_{i}^{*}$ $\supset X \cup Y) \subset X \dagger \cup Y \dagger$, since the latter under the distributive law appears as an intersection of finite $\sum Q_{i}{ }^{*} \supset X \cup Y$.

We now prove $Q \dagger=Q^{*}$ for any subgroup $Q$, from which (a) and (b) follow at once. By definition, $Q \dagger \subset Q^{*}$. But since $Q \dagger=\cap\left(\sum Q_{i}^{*} \supset Q\right)$, by (c) $\sum Q_{i}^{*} \supset Q$ if and only if $\sum Q_{i}^{*} \supset Q^{*}$. Hence $Q \dagger=Q^{*} \dagger \supset Q^{*}$.

THEOREM 13. In order that there exist a C1-4, C7 (topological) closure $X \rightarrow X \dagger$ on the subsets of $\Omega$, such that (a) and (b) of Theorem 12 obtain, it is necessary and sufficient that

(d) for groups $Q, Q_{1}, \cdots, Q_{n}$ and elements $q_{1}, \cdots, q_{m}$, either $n$ or $m$ of which may be zero, $Q \subset Q_{1}{ }^{*} \cup \ldots \cup Q_{n}{ }^{*} \cup q_{1} \cup \ldots \cup q_{m}$ implies $Q^{*} \subset Q_{1}{ }^{*} \cup \ldots \cup Q_{n}{ }^{*}$ $\cup q_{1} \cup \ldots \cup \cup_{m}$.

Necessity. As before, we prove $Q i=Q^{*}$ for a group $Q$, and $Q^{*} \dagger=Q^{*}$. Hence if the hypothesis of (d) holds, we have $Q^{*}=Q \dagger \subset Q_{1}{ }^{*} \uparrow \cup \ldots \cup Q_{1}{ }^{*} \nmid \cup q_{1} \dagger \cup \ldots$ $\cup q_{m} \dagger=Q_{1}{ }^{*} \cup \ldots \cup Q_{n}{ }^{*} \cup q_{1} \cup \ldots \cup q_{m}$.

Sufficiency. Define $X \dagger$ as the set intersection of all finite set unions $Q_{1}^{*} \cup \ldots \cup Q_{n}{ }^{*} \cup q_{1} \cup \ldots \cup \cup_{q_{m}}$ containing $X$ (either $n$ or $m$ may be zero). We verify C1-4 as before, and obviously $p \dagger=p$ hence C7. Again (a) and (b) follow from the result that $Q^{*}=Q \dagger$ for a subgroup $Q$. The proof is just as above.

COROllary 6. For a topologization $\mathrm{C} 1-4,7$ of a $\left(^{*}\right)$ closure in $\Omega$, it is necessary that $Q^{*}=Q$ for a finite subgroup $Q$. 
For we can write $Q=q_{1} \cup \ldots \cup q_{m}$ and condition (d) implies $Q^{*} \subset q_{1}$ $\cup \ldots \cup q_{m}=Q$.

We remark that the Krull topology satisfies $\mathrm{C} 1-4,7$, (a) and (b) and hence (d'). (a) holds because the topological closure of a subgroup of a topological group is a group.

8. On primary abelian groups. Let $\Pi$ be a primary abelian group of characteristic $\pi$, a prime number, with total (proper) automorphism group $\Omega$. The binary relation $p \rho q$ meaning $q(p)=p$ on elements $p \in \Pi, q \in \Omega$ defines a galois correspondence $\mathfrak{P}_{\rho}(Q)$ and $\mathfrak{Q}_{\rho}(P)$ on $Q \subset \Omega, P \subset \Pi$ in the usual way (cf: $\S 5$ ). This induces a galois correspondence on the subgroup lattices $\mathfrak{B}$ of $\Pi$ and $\mathfrak{Q}$ of $\Omega$. Denote by $\mathfrak{F}$ the set of all subgroups $F$ of $\Pi$ the periods of whose elements are bounded.

THEOREM 14. The set $\mathfrak{F}$ of subgroups of elements of bounded periods satisfies F1, 2 of Theorem 9 and the condition of Theorem 10. The corresponding Krull topology converts $\Omega$ into a zero-dimensional, metrizable topological group.

For $\mathfrak{F} 1$ is obvious, and $\mathfrak{F} 2$ is valid since every subgroup $P \subset \Pi$ is a union of the cyclic groups of all its elements. Let $F_{n}$ be the (characteristic) group of all elements whose period is less than or equal to $\pi^{n}$. This sequence satisfies the condition of Theorem 10. The theorem follows from Theorems 9, 10 and Corollary 4.

However examples show $\mathfrak{F} 3$ fails and this topology fails to characterize the galois closed subgroups $Q=\mathfrak{Q} \Re Q$. For non-topological characterization, see Shiffman [13].

The topology of Theorem 13 should be compared with an analogous but different topology introduced by G. Birkhoff [4]. We make no further attempt to discuss topologies for $\Omega$, but show how Theorem 12 combined with results of Baer [2] throws light on the galois closed subgroups $P=\mathfrak{B Q} P$ of $\Pi$.

We quote briefly the results of Baer which we use below. Define $\pi^{i} \Pi=$ (all $\left.\pi^{i} p ; p \in \Pi\right)$, and $\pi^{\omega} \Pi=\cap\left(\pi^{i} \Pi, i=1,2, \cdots\right)$. Define $p^{\infty} \Pi=$ maximal subgroup $P$ such that $\pi P=P$. If $\pi \neq 2$, then $\mathfrak{B Q}(0)=0$. For if $\mathfrak{B Q}(0) \supset p \neq 0$, $p$ is left fixed by every automorphism in $\mathfrak{Q}(0)=\Omega$. Since $p \rightarrow-p$ is an automorphism we have $p=-p, 2 p=0, \pi=2$.

If $\pi^{\omega} \Pi=0$ and $\pi \neq 2$, then $\mathfrak{B Q}(P) / P=\pi^{\omega}(\Pi / P)$. If $\pi \neq 2, \mathfrak{B Q}(P)=P$ for all $P \subset \Pi$ if and only if either $\pi^{\infty} \Pi \neq 0$ or the periods of the elements of $\Pi$ are bounded.

We now prove the following theorem.

THEOREM 15. If $\Pi$ is a primary abelian group with $\pi \neq 2$ and $\pi^{\omega} \Pi=0$, then condition (c) of Theorem 12 holds for subgroups $P \subset \Pi$, and the closure $P \dagger$ there defined satisfies C1-4. A subgroup $P$ is galois closed, $P=\mathfrak{P Q}(P)$, if and only if it is $(\dagger)$ closed.

Let $P \subset P_{1}^{*} \cup \cdots \cup P_{n}^{*}$ where $P^{*}$ means $\mathfrak{B Q}(P)$, where $P, P_{1}, \cdots, P_{n}$ 
are subgroups of $\Pi$. By Baer's result, $P^{*} / P=\pi^{\omega}(\Pi / P)$, that is, $P^{*}=$ $\cap\left(\pi^{i}(\Pi / P)\right)$. Let $p_{0} \in P^{*}$. Then coset $p_{0}+P \in \pi^{i}(\Pi / P)$, for all $i$. Write $p_{0}+P=\pi^{i}\left(p_{i}+P\right)$ and $p_{0}=\pi^{i} p_{i}+p_{i}^{\prime}, p_{i}^{\prime} \in P, i=1,2, \cdots$. Since $p_{i}^{\prime} \in P$ $\subset P_{1}^{*} \cup \ldots \cup P_{n}^{*}$, an infinite subsequence $p_{i_{k}}^{\prime}$ must fall in one of the $P_{i}^{*}$, say $P_{1}^{*}$. Thus $p_{0}=\pi^{i k} p_{i k}+p_{i_{k}}^{\prime}, p_{0}+P_{1}^{*}=\pi^{i k}\left(p_{i k}+P_{1}^{*}\right)$. Since $\pi\left(\Pi / P_{1}^{*}\right)$ $\supset \pi^{2}\left(\Pi / P_{1}^{*}\right) \supset \cdots$ we must have $p_{0}+P_{1}^{*} \in \pi^{i}\left(\Pi / P_{1}^{*}\right)$, for all $i$, and $p_{0}+P_{1}^{*}$ $C \pi^{\omega}\left(\Pi / P_{1}^{*}\right)=[0]$ since $P_{1}^{*}$ is galois-closed. Hence $p_{0}+P_{1}^{*}=P_{1}^{*}$ and $p_{0} \in P_{1}^{*}$. We have thus $P^{*} \subset P_{1}^{*} \cup \ldots \cup P_{n}^{*}$.

9. Topologization theorems. Although the theorems of $\$ 7$ were stated with reference to the subgroup lattice of a group, for convenience in discussing the applications, no use was made of the group properties. We have really proved the following theorems.

THEOREM 16. Let $\Omega$ be a set of elements $q, \mathfrak{Q}$ any partially ordered set of subsets $Q \subset \Omega$ containing $\Omega$ and closed under $\cap$, which admits a $\mathrm{C} 1-3$ closure $Q \rightarrow Q^{*}$. In order that there exist a C1-4 closure $X \rightarrow X \dagger$ on the subsets $X \subset \Omega$ such that, for $Q \in \mathbb{Q}$,

(a) $Q \dagger \in \mathbb{Q}$,

(b) $Q \dagger=Q$ if and only if $Q^{*}=Q$,

it is necessary and sufficient that

(c) for $Q, Q_{1}, \cdots, Q_{n} \in Q, Q \subset Q_{1}^{*} \cup \cdots \cup Q_{n}^{*}$ implies $Q^{*} \subset Q_{1}^{*} \cup \cdots \cup Q_{n}{ }^{*}$.

THEOREM 17. In order that there exist a $\mathrm{C} 1-4, \mathrm{C7}$ (topological) closure $X \rightarrow X \dagger$ on the subsets $X \subset \Omega$ such that (a), (b) of Theorem 16 hold for $Q \in \mathfrak{Q}$, it is necessary and sufficient that

(d) for $Q, Q_{1}, \cdots, Q_{n} \in \mathfrak{Q}, q_{1}, \cdots, q_{m} \in \Omega$ (either $n$ or $m$ may be 0 ), $Q \subset Q_{1}^{*} \cup \ldots \cup Q_{n} \cup_{q_{1}} \cup \ldots \cup \cup_{q_{m}}$ implies $Q^{*} \subset Q_{1}^{*} \cup \ldots \cup Q_{n}^{*} \cup q_{1} \cup \ldots$ $\cup q_{m}$.

\section{BiBLIOGRAPHY}

1. L. Alaoglu, Weak topologies of normed linear spaces, Ann. of Math. (2) vol. 41 (1940) pp. 252-267.

2. R. Baer, Primary abelian groups and their automorphisms, Amer. J. Math. vol. 59 (1937) pp. 99-117.

3. S. Banach, Theorie des operations lineaires, Warsaw, 1932.

4. G. Birkhoff, Hausdorff groupoids, Ann. of Math. (2) vol. 35 (1934) pp. 351-360.

5. - , On the structure of abstract algebras, Proc. Cambridge Philos. Soc. vol. 31 (1935) pp. 433-454.

6. - Lattice theory, Amer. Math. Soc. Colloquium Publications, vol. 25, 1940.

7. A. H. Frink, Distance functions and the metrization problem, Bull. Amer. Math. Soc. vol. 43 (1937) pp. 133-142.

8. W. Krull, Galoissche Theorie der unendlichen algebraischen Erweiterungen, Math. Ann. vol. 100 (1928) pp. 687-698.

9. H. MacNeille, Partially ordered sets, Trans. Amer. Math. Soc. vol. 42 (1937) pp. 416-460.

10. E. H. Moore, Introduction to a form of general analysis, Amer. Math. Soc. Colloquium Publications, vol. 2, 1910. 
11. O. Ore, Mathematical relations and structures, Amer. Math. Soc. Colloquium Lecture, Cf. Bull. Amer. Math. Soc. vol. 48 (1942) pp. 169-182.

12. H. Prüfer, Untersuchungen über Teilbarkeitseigenschaften in Körpern, J. Reine Angew. Math. vol. 168 (1932) pp. 1-36.

13. M. Shiffman, The ring of automorphisms of an abelian group, Duke Math. J. vol. 6 (1940) pp. 579-597.

14. H. Terasaka, Theorie der topologischen Verbände, Proc. Imp. Acad. Tokyo vol. 13 (1937) pp. 401-405.

15. S. Ulam and C. J. Everett, Projective algebra, to appear in Proc. Nat. Acad. Sci.U.S.A.

16. M. Ward, Closure operators of a lattice, Ann. of Math. (2) vol. 43 (1942) pp. 191-196.

UNIVERSITY OF WISCONSIN,

Madison, Wis. 